\title{
Risk factors distribution and cardiovascular disease prevalence in the Italian population: The CHECK study
}

\author{
Elena Tragni ${ }^{1}$, Alessandro Filippi ${ }^{2}$, Manuela Casula ${ }^{1}$, Giampiero Favato ${ }^{3}$, Ovidio Brignoli ${ }^{2}$, \\ Claudio Cricelli ${ }^{2}$, Andrea Poli ${ }^{1}$, Alberico L. Catapano ${ }^{1,4^{*}}$ (for the CHECK Group ${ }^{\#}$ )
}

\author{
${ }^{1}$ Epidemiology and Preventive Pharmacology Centre (SEFAP), University of Milano, Milano, Italy \\ ${ }^{2}$ Italian Society of General Medicine (SIMG), Firenze, Italy \\ ${ }^{3}$ Kingston University, Kingston upon Thames, UK \\ ${ }^{4}$ IRCCS Multimedica, Sesto S. Giovanni, Milano, Italy \\ Email: ${ }^{*}$ alberico.catapano@unimi.it
}

Received 5 August 2012; revised 15 September 2012; accepted 8 December 2012

\begin{abstract}
Objective: To evaluate the distribution of cardiovascular risk factors and the prevalence of cardiovascular disease in a sample of the Italian population. Methods: CHECK (Cholesterol and Health: Education, Control and Knowledge) is a cross-sectional observational study in a randomised sample of the Italian adult population aged 40 - 79 years, in the setting of general practice. Results: 5846 subjects $(50.3 \%$ male) were included in the analysis. The mean age $[ \pm \mathrm{SD}]$ of the observed cohort was $57.8( \pm 10.3)$ years. One out of five subjects smoked cigarettes and almost $80 \%$ didn't engage in regular leisure-time physical activity. The mean blood pressure was $132.0[ \pm \mathbf{1 4 . 7 ] / 8 1 . 2}[ \pm 7.9]$ mmHg. The total and LDL-cholesterol levels were respectively $205.3[ \pm 35.9] \mathrm{mg} / \mathrm{dL}$ and $124.9[ \pm 29.9]$ $\mathrm{mg} / \mathrm{dL}$. The mean glucose concentration was 98.3 $[ \pm 28.2] \mathrm{mg} / \mathrm{dL}$. The prevalence rate of hypertension, hypercholesterolemia, and type 2 diabetes were respectively $51.8 \%, 55.6 \%$, and $13.0 \%$. $8.9 \%$ of the observed subjects had a history of cardiovascular events, while in the primary prevention group the 10-yearrisk of coronary heart disease (Framingham algorithm) was $10.1 \%[ \pm 8.3 \%]$ and of cardiovascular disease (CUORE algorithm) was $5.2 \%$ [ $\pm 5.9 \%]$. Conclusion: The CHECK study provides a detailed description of a randomised sample of the Italian population, contributing to evaluate the prevalence of cardiovascular risk factors and the main cardiovascular disease in Italy and to provide a baseline to set priorities and objectives for future intervention of health policy.
\end{abstract}

Keywords: Italian Population; Epidemiological Study;

*Corresponding author.

\#As presented in Appendix.
Cardiovascular Risk Factors; Prevalence of

Cardiovascular Disease

\section{INTRODUCTION}

Cardiovascular diseases (CVD), a group of disorders of the heart and blood vessels, including coronary heart disease, cerebrovascular disease, peripheral arterial disease, are the leading cause of morbidity, disability and mortality worldwide, showing a continue increasing trend. In 2008, CVD caused 17 million deaths $(47.2 \%$ of noncommunicable diseases deaths, $29.8 \%$ of total deaths in the world) [1]; in particular, 7.6 million deaths were due to ischemic heart disease and 5.7 million to stroke [2].

CVD remains the first cause of death and disability in Italy although a slow and stepwise reduction of incidence has been observed since the mid seventies [3,4].

The subjects who survive acute cardiovascular events are likely to become chronic patients. CVD compromise patients' quality of life and represent a significant economic burden to the national health system. Cardiovascular drugs account for about $26.4 \%$ of the Italian Pharmaceutical expenditure [5].

The CHECK (Cholesterol and Health: Education, Control and Knowledge) study was designed to provide information on the distribution of the main CVD risk factors in Italy setting the stage for addressing questions relative to the plausibility and feasibility of interventions on the principal risk factors for CVD in an European country.

CHECK is the first randomised Italian epidemiological study performed in primary care. Its main objective was to evaluate the distribution of cardiovascular risk factors and the prevalence of cardiovascular disease in a representative sample of the Italian adult population aged 40 - 79 years. Other aims were 1) to perform a follow-up study to determine the incidence of cardiovascular events 
and other main pathologies, 2) to create plasma and DNA banks to design ad-hoc nested case-control studies, 3) to disseminate information about cardiovascular risk factors to increase physicians and patients awareness and contribute to the implementation of evidences supporting health policies and preventive strategies in the population.

In this paper we describe baseline characteristics of enrolled subjects.

\section{MATERIAL AND METHODS}

The study was conducted in accordance to the Declaration of Helsinki, Guidelines for Good Clinical practice, and the Italian bioethics regulations and laws. The study was approved by the local Ethic Committee and all subjects gave written informed consent to the study (protocol code SEFAP/Pr. 0003).

\subsection{Study Design}

The Italian Society of General Medicine (SIMG) identified 54 general practitioners (coordinators) that were active at local level and distributed throughout the Italian territory (both rural and urban areas) in proportion to the regional population density. Each coordinator had to contact other general practitioners (GPs) who were operating on the same territory, to reach a group of 14 GPs.

Overall, 764 GPs were invited to participate to the study as investigators. They had to meet all the following pre-requisites:

- age: $<52$ years;

- practice size: $>1000$ subjects;

- computerised patient records system;

- access to the web.

The GPs who adhered to the proposal had to registered in the project website and to provide some personal information and their patients number. Each investigator was required to enrol 16 subjects aged 40 to 79 years, randomly selected among his patients, following a random number list generated by the coordinating Centre in Milan based on self-reported number of patients. The patient selection procedure was subject to random control by the local coordinators. Subjects who didn't give their consensus or who were not eligible for practical reasons (e.g. subjects with walking disabilities, severe handicap, or suffering from an invaliding disease or a severe mental illness) were excluded from the randomized sample. The subjects' enrolment started in March 2002 and ended in June 2005.

\subsection{Data Collection}

Subjects' clinical history and clinical information were collected during a standardised visit performed by the investigating physician.

Information about smoking habit, physical activity, alcohol use and chronic drug treatments were collected directly from the patient during the same examination. Smoking habit was classified as current (patient smoking at least one cigarette per day) or former (at least one year from smoking cessation). Self-reported leisure-time physical activity was classified as present or non-present. The use of alcohol was evaluated by adding the consumption of red and/or white wine, beer and liquors. Information on chronic therapies was also collected.

Weight and height measures, obtained from lightly clothed patients, were expressed in terms of body mass index (BMI, weight in kilos divided by the square value of height, in meters).

The measurement of blood pressure (BP) was performed with the patient in sitting position, after 4 minute of rest, applying the appropriate cuff on the right arm. Systolic and diastolic blood pressure was identified at the beginning of the first and the fifth phase of the Korotkoff sounds, using a mercury sphygmomanometer with the appropriate cuff. Three consecutive readings were recorded and their mean was considered for the analysis. Heart rate was assessed as beats per minute after the last measurement of BP.

The presence of angina pectoris or of a history of myocardial infarction, coronary artery bypass graft or coronary angioplasty, stroke, transient ischemic attacks, claudicatio and left ventricular hypertrophy were assessed by retrieving the medical records of the subject and by direct inquiry. Subjects with a history of cardiovascular events (excluding left ventricular hypertrophy) were considered in secondary prevention.

Family history for premature cardiovascular diseases was determined when episodes of stroke and/or myocardial infarction were reported for one or more first-degree relatives, $<55$ years old for men and $<65$ years old for women. Family history for hypertension, dislipidemia, and diabetes was also evaluated.

All relevant subject records were entered in a computerized patient chart directly by the investigating physician and then sent to a central server via web.

Blood samples were drawn at the beginning of the enrolment visit. Blood was obtained between 8 to $10 \mathrm{am}$, from the antecubital vein, in sitting position, after 12 hours of fasting and alcohol abstinence. Blood samples were then collected in EDTA or monoiodine-acetate (only for glucose assessment) coated tubes and shipped by courier at $4^{\circ} \mathrm{C}$ temperature to the central laboratory (Fleming $\mathrm{SpA}$, in Brescia, Italy) within 24 hours, where the biochemical parameters were determined. The biochemical evaluation was performed following the criteria of the World Health Organization Lipid Reference Laboratories. 
Upon arrival, the samples were centrifuged to obtain the plasma. Levels of total cholesterol (TC), glucose and triglycerides (TG) were measured by a chromatometric enzymatic method; the plasma levels of apolipoprotein B by a latex enhanced turbidimetric immuno-assay. Plasma HDL cholesterol levels (HDL-C) were measured after the precipitation of the apolipoprotein $\mathrm{B}$ containing lipoproteins with dextran-magnesium-chloride. All determinations were performed by an automatic analyzer (ADVIA 1650; Bayer, Germany). Fibrinogen plasma levels $(1.8-3.5 \mathrm{~g} / \mathrm{L})$ were assessed by a turbidimetric method with Nefelometro BNA 100 (Boehring, Italia). The low density lipoprotein cholesterol (LDL-C) values were calculated using the Friedewald formula (TC minus HDL-C minus $1 / 5 \mathrm{TG})$.

Serum $1 \mathrm{~mL}$ aliquots (4 - 5 for each sample) were frozen at $-80^{\circ} \mathrm{C}$ for further biochemical evaluation. White cells were frozen at $-20^{\circ} \mathrm{C}$ for polymorphism search/ genetic research.

Criteria used for specific diagnosis were:

- Hypercholesterolemia: plasma levels of $\mathrm{TC} \geq 200$ $\mathrm{mg} / \mathrm{dL}$ or LDL-C $\geq 130 \mathrm{mg} / \mathrm{dL}$ or pharmacological treatment with statins and/or simvastatin + ezetimibe.

- Hypertriglyceridemia: plasma levels of TG $\geq 170 \mathrm{mg} /$ $\mathrm{dL}$ or pharmacological treatment with fibrates and/or omega-3.

- Mixed dyslipidemia: hypercholesterolemia and hypertriglyceridemia, as defined above.

- Hypertension: recorded diagnosis by physician or systolic BP $\geq 140 \mathrm{~mm} \mathrm{Hg}$ or diastolic BP $\geq 90 \mathrm{~mm}$ $\mathrm{Hg}$ or currently taking medication to lower high BP (NHANES criteria [6]).

- Type 2 diabetes mellitus: recorded diagnosis by physician or fasting blood glucose levels $\geq 126 \mathrm{mg} / \mathrm{dL}$ or currently taking antidiabetic drugs (oral hypoglycaemic medication and/or insulin).

- Overweight: body mass index $(\mathrm{BMI}) \geq 25$ and $<30$ $\mathrm{kg} / \mathrm{m}^{2}$;

- Obesity: BMI $\geq 30 \mathrm{~kg} / \mathrm{m}^{2}$;

- Metabolic Syndrome (MetS): presence of at least 3 of 5 of the listed characteristics [7]: abdominal obesity, given as waist circumference, men $>102 \mathrm{~cm}$, women $>88 \mathrm{~cm}$; triglycerides $\geq 150 \mathrm{mg} / \mathrm{dL}$; HDL cholesterol, men $<40 \mathrm{mg} / \mathrm{dL}$, women $<50 \mathrm{mg} / \mathrm{dL}$; blood pressure $\geq 130 / 85 \mathrm{mmHg}$; fasting glucose $\geq$ $110 \mathrm{mg} / \mathrm{dL}$. BMI was used as a surrogate of waist circumference, men $\geq 28 \mathrm{~kg} / \mathrm{m}^{2}$, women $\geq 25 \mathrm{~kg} / \mathrm{m}^{2}$ (approach validated in a subgroup of about $1000 \mathrm{sub}-$ jects: assignment to MetS/noMetS consistent in $92 \%$ of cases in males and $97 \%$ in females).

The coronary heart disease risk (CHD risk \% in 10 years) was calculated using the Framingham algorithm [8], as recommended at the time of the study by the Italian regulatory Agency (AIFA) for the prescription of statin drugs [9]; the global cardiovascular risk (CVD risk $\%$ in 10 years) was evaluated using the CUORE algorithm [10], developed from epidemiological data obtained in Italy, as reported by Italian recommendations since 2003 [11]. Based on Framingham or CUORE scores, subjects were stratified in four risk classes $(<5 \%$ low-risk; $5 \%$ - 10\% mild-risk; $10 \%$ - 20\% moderaterisk; $\geq 20 \%$ high risk).

\subsection{Statistical Analysis}

Normally distributed variables are presented as mean values $[ \pm$ standard deviation, SD] and not normally distribute variables as median [interquartile range, IQR], while qualitative variables are presented as frequencies. Comparisons between continuous variables across sex groups were performed by using the t-test for independent sample. Comparisons between proportions across sex groups for categorical (and qualitative) variables were performed by using non-parametric tests. All reported p-values are based on two-sided tests and compared to a significance level of 5\%.

All statistical analyses were performed using the Windows 16.0 version of SPSS (SPSS, Inc., Chicago, Illinois).

\section{RESULTS}

432 GPs participated to the CHECK study (mean age 47.8 years, $80.1 \%$ male; $56.5 \%$ participation rate). Each of them enrolled up to 16 subjects, following the randomised sampling procedure. Overall, 6890 subjects were enrolled; 1044 (15.1\%) of them did not attend the medical visit and were excluded from the study sample; the blood samples of 131 subjects $(1.9 \%)$ were not processable, although their anamnestic data were included in the analysis.

5846 subjects were included in the final analysis (Figure 1). Men were $49.7 \%$ of the cohort. The mean age was 57.8 years (58.2 years for men and 57.4 for women). Comparison between the Check demographics and the latest available census data [12] showed small difference: women were slightly under-represented in the CHECK sample (50.3\% vs. $52.6 \%$ respectively); the $50-59$ and 60 - 69 age groups, conversely, were slightly over-represented $(30.5 \%$ vs. $27.0 \%$ and $28.0 \%$ vs. $24.6 \%$, respectively).

The main outcomes of the study are shown in Tables 1-4. The average level of education is quite low: $33.0 \%$ of subjects achieved only primary education, but this percentage was higher in women (36.7\% vs. $29.2 \%)$. $32.9 \%$ reported to be retired, with a high proportion of men $(65.8 \%) ; 23.3 \%$ were housewives $(45.8 \%$ of the female sample). Smoke habit and alcohol consumption were more prevalent in men $(24.3 \%$ vs. $18.6 \%$ and $62.4 \%$ vs. $28.4 \%$, respectively), whereas physical inac- 


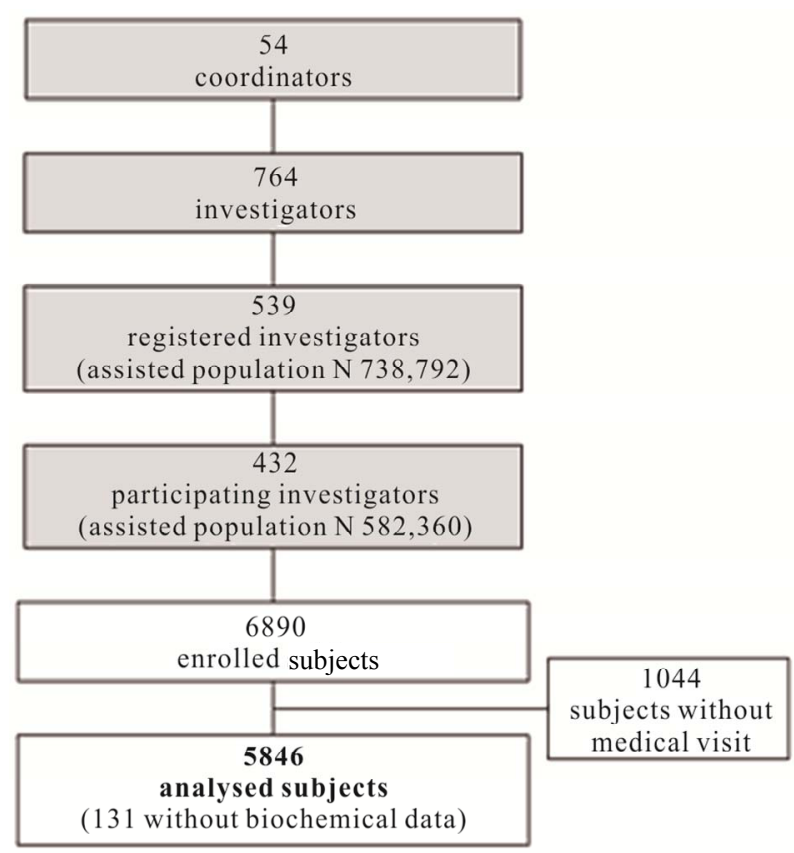

Figure 1. Flow chart of the study.

tivity was more present in women $(84.1 \%$ vs. $75.0 \%)$ (Table 1).

Mean BMI $\left( \pm\right.$ SD) was $26.5 \pm 4.3 \mathrm{~kg} / \mathrm{m}^{2}$, putting the sample in the overweight range; this was observed in both genders. Mean SBP/DPB values were (132.0 \pm 14.7)/(81.2 \pm 7.9$) \mathrm{mm} \mathrm{Hg}$, slightly higher than cut off for pre-hypertension. Mean total cholesterol was $205.3 \pm$ $35.9 \mathrm{mg} / \mathrm{dL}$, while LDL-cholesterol was $124.9 \pm 29.9$ $\mathrm{mg} / \mathrm{dL}$, the former just higher and the latter slightly lower than desirable levels, due to HDL-cholesterol values $(54.8 \pm 12.2 \mathrm{mg} / \mathrm{dL})$ (Table 2$)$.

As shown in Table 3, 51.8\% of subjects were hypertensive, $13.0 \%$ were diabetic, and $55.6 \% / 20.8 \%$ were hypercholesterolemic/hypertriglyceridemic. $8.6 \%$ had a history of cardiovascular events and thus could be defined as in secondary $\mathrm{CV}$ prevention. The $65.0 \%$ of the remaining subjects had a global CV risk in 10 years (calculated with CUORE algorithm) lower than 5\%, with a remarkable difference between genders (mean values: $7.8 \% \pm$ $7.1 \%$ in men vs. $2.8 \% \pm 3.1 \%$ in women) (Table 4 ).

\section{DISCUSSION}

The main objective of the CHECK study was to evaluate the cross-sectional prevalence of CVD and cardiovascular risk factors in a cohort of randomly selected Italian subjects, aged 40 to 79 years.

The CHECK sample proved to be representative of the Italian population in the same age range. The differences observed by comparing the Check demographics to the latest available census data [12] are small, and of limited relevance. This was probably due to a larger participation to the study of subjects in a non-working age or condition.

The CHECK study provided a detailed and in depth description of the prevalence of cardiovascular risk factors and the main cardiovascular pathologies in the Italian population. In order to put our findings into a wider context, we compare them with other national surveys, while maintaining a worldwide perspective.

Smoking is estimated to cause nearly $10 \%$ of cardiovascular disease (5.4 millions of deaths) [13]. Currently, there are about 1 billion smokers worldwide [14]. The proportion of smoking subjects in the CHECK sample was $21.4 \%$ (men $24.3 \%$ and women $18.6 \%$ ). These figures were slightly lower than the official census data (24.5\% in 2003, 22.6\% in 2004) [15]; the Check cohort excluded the 25 - 34 age group, the one with the highest prevalence of smokers $(31.4 \%)$, especially among men. The reduced prevalence of smokers in the CHECK cohort, as compared to previous epidemiological data [16], could also reflect the declining smoking habits in the Italian population, as a result of educational programs and smoking restrictions enforced in public buildings.

Physical inactivity is one of the leading risk factors for mortality and is estimated to cause about $30 \%$ of ischaemic heart disease burden [13]. Globally, around $31 \%$ of adults are insufficiently active (men $28 \%$ and women $34 \%$ ) [17]. Concerning leisure-time physical activity, the self-reported information obtained from the CHECK cohort were less favourable than the data derived from other studies. The prevalence of physical inactivity was $75 \%$ in men and $84 \%$ in women, vs. $34 \%$ in men and $46 \%$ in women observed, as an example, in the Atlante study [18].

Overweight and obesity lead to adverse metabolic effects on blood pressure, cholesterol, triglycerides and insulin resistance [13]; all over the world, 2.8 million people die each year as a result of being overweight [13]. A total of more than half a billion adults worldwide are obese [19]. The mean BMI of the CHECK cohort was $26.5 \mathrm{~kg} / \mathrm{m}^{2}$, well within the overweight range. $17.4 \%$ of the cohort subjects were obese, with a higher prevalence in women than in men ( $18.8 \%$ vs. $16.0 \%$, respectively). In the PASSI study, a national survey (self-reported information) conducted on younger people aged 18 - 69 years [20], 32\% of Italians are overweight, while one in ten is obese [21].

Raised blood pressure is the leading risk factor for cardiovascular disease mortality, causing more than 7 million deaths every year worldwide. Globally, prevalence of hypertension in adults is around $40 \%$ [13]. In our study, the mean blood pressure value was 132.0/81.2 $\mathrm{mmHg}$. Hypertension was present in more than half of the sample, with a higher prevalence in men $(54.1 \% \mathrm{M}$ vs. $49.5 \% \mathrm{~W})$. The CHECK mean SBP values were comparable to those obtained in other Italian observational 
Table 1. Sociodemographic and lifestyle characteristics in total and sex-stratified sample.

\begin{tabular}{|c|c|c|c|}
\hline & Total sample & Men & Women \\
\hline Subjects, n (\%) & 5846 & 2903 (49.7) & $2943(50.3)$ \\
\hline Age, mean $\pm \mathrm{SD}\left(^{*}\right)$ & $57.8 \pm 10.3$ & $58.2 \pm 10.1$ & $57.4 \pm 10.5$ \\
\hline \multicolumn{4}{|c|}{ Age distribution, $\mathrm{N}(\%)^{\S}$} \\
\hline 40 - 49 years & $1517(26.0)$ & $689(23.7)$ & $828(28.1)$ \\
\hline 50 - 59 years & $1784(30.5)$ & $912(31.4)$ & $872(39.6)$ \\
\hline 60 - 69 years & $1638(28.0)$ & $844(29.1)$ & $794(27.0)$ \\
\hline 70 - 79 years & $907(15.5)$ & $458(15.8)$ & $449(15.3)$ \\
\hline \multicolumn{4}{|c|}{ Occupational status, $\mathrm{N}(\%)^{\S}$} \\
\hline Employed & $2109(36.5)$ & $1360(47.4)$ & 749 (25.8) \\
\hline Housewife & $1347(23.3)$ & $14(0.5)$ & $1333(45.8)$ \\
\hline Retiree & $1902(32.9)$ & $1252(43.7)$ & $650(22.3)$ \\
\hline Unemployed & $51(0.9)$ & $33(1.2)$ & $18(0.6)$ \\
\hline Other & $367(6.4)$ & $207(7.2)$ & $160(5.5)$ \\
\hline \multicolumn{4}{|c|}{ Educational level (highest degree), $\mathrm{N}(\%)^{\S}$} \\
\hline None & $98(1.7)$ & $37(1.3)$ & $61(2.1)$ \\
\hline Elementary & $1905(33.0)$ & $836(29.2)$ & $1069(36.7)$ \\
\hline Junior high & 1840 (31.9) & $916(32.0)$ & $924(31.8)$ \\
\hline High school & $1488(25.8)$ & $808(28.2)$ & $680(23.4)$ \\
\hline University & $445(7.7)$ & $269(9.4)$ & $176(6.0)$ \\
\hline \multicolumn{4}{|c|}{ Life habits, N $(\%)^{\S}$} \\
\hline Smokers & $1253(21.4)$ & $706(24.3)$ & $547(18.6)$ \\
\hline Regular exercise & $1181(20.4)$ & $717(25.0)$ & 464 (15.9) \\
\hline Alcohol users & $2613(45.2)$ & $1788(62.4)$ & $825(28.4)$ \\
\hline
\end{tabular}

${ }^{*}$ Men vs. women (t-test) $\mathrm{p}=0.003 ;{ }^{\S}$ Men vs. women (non-parametric tests) $\mathrm{p}<0.05$.

Table 2. Anthropometric, vital and biochemical parameters in total and sex-stratified sample, mean \pm SD.

\begin{tabular}{|c|c|c|c|c|}
\hline & & Total sample & Men & Women ${ }^{*}$ \\
\hline Height (m) & $\mathrm{N}=5843$ & $1.65 \pm 0.09$ & $1.71 \pm 0.07$ & $1.60 \pm 0.07$ \\
\hline Weight (kg) & $\mathrm{N}=5835$ & $72.5 \pm 13.4$ & $78.5 \pm 11.7$ & $66.6 \pm 12.4$ \\
\hline BMI $\left(\mathrm{Kg} / \mathrm{m}^{2}\right)$ & $\mathrm{N}=5835$ & $26.5 \pm 4.3$ & $26.9 \pm 3.5$ & $26.2 \pm 4.9$ \\
\hline SBP (mmHg) & $\mathrm{N}=5844$ & $132.0 \pm 14.7$ & $133.0 \pm 13.8$ & $130.9 \pm 15.5$ \\
\hline DBP $(\mathrm{mmHg})$ & $\mathrm{N}=5844$ & $81.2 \pm 7.9$ & $81.9 \pm 7.8$ & $80.6 \pm 8.0$ \\
\hline Heart rate (bpm) & $\mathrm{N}=5773$ & $73.3 \pm 8.5$ & $72.6 \pm 8.5$ & $74.0 \pm 8.5$ \\
\hline TC $(\mathrm{mg} / \mathrm{dL})$ & $\mathrm{N}=5710$ & $205.3 \pm 35.9$ & $202.5 \pm 35.7$ & $208.0 \pm 35.9$ \\
\hline HDL-C (mg/dL) & $\mathrm{N}=5710$ & $54.8 \pm 12.2$ & $50.7 \pm 10.4$ & $58.8 \pm 12.4$ \\
\hline TG $(\mathrm{mg} / \mathrm{dL})$ & $\mathrm{N}=5710$ & $132.8 \pm 102.1$ & $149.9 \pm 120.6$ & $115.9 \pm 76.1$ \\
\hline median [IQR] & & $109[78-157]$ & 119 [86 - 177] & $98[72-140]$ \\
\hline LDL-C $(\mathrm{mg} / \mathrm{dL})^{\S}$ & $\mathrm{N}=5614$ & $124.9 \pm 29.9$ & $123.3 \pm 29.3$ & $126.5 \pm 30.3$ \\
\hline ApoB (mg/dL) & $\mathrm{N}=5710$ & $111.4 \pm 25.2$ & $112.5 \pm 25.3$ & $110.4 \pm 25.1$ \\
\hline Non-HDL-C $(\mathrm{mg} / \mathrm{dL})^{\#}$ & $\mathrm{~N}=5710$ & $150.5 \pm 33.8$ & $151.8 \pm 33.6$ & $149.2 \pm 34.0$ \\
\hline Glucose (mg/dL) & $\mathrm{N}=5547$ & $98.3 \pm 28.2$ & $101.7 \pm 29.6$ & $94.9 \pm 26.3$ \\
\hline median [IQR] & & $92[84-102]$ & 95 [87 - 106] & 89 [83 - 98] \\
\hline Fibrinogen $(\mathrm{g} / \mathrm{L})$ & $\mathrm{N}=5710$ & $3.41 \pm 0.69$ & $3.34 \pm 0.70$ & $3.47 \pm 0.68$ \\
\hline
\end{tabular}

BMI: body mass index; SBP: systolic blood pressure; DBP: diastolic blood pressure; TC: total cholesterol; TG: triglycerides; apoB: apolipoprotein B; ${ }^{*}$ Men vs women (t-test) $\mathrm{p}<0.01$ for all comparisons; ${ }^{\$}$ Obtained by the Friedewald formula; ${ }^{*}$ (TC) - (HDL-C). 
Table 3. Prevalence of cardiovascular disease and related comorbidities in total and sex-stratified sample, N (\%).

\begin{tabular}{|c|c|c|c|c|c|}
\hline & & Total sample & Men & Women & $\mathbf{p}^{*}$ \\
\hline \multicolumn{6}{|c|}{ Comorbidities } \\
\hline Hypertension & $\mathrm{N}=5774$ & $2990(51.8)$ & $1549(54.2)$ & $1441(49.5)$ & $<0.001$ \\
\hline Hypercholesterolemia & $\mathrm{N}=5707$ & $3172(55.6)$ & $1514(53.5)$ & $1661(57.9)$ & $<0.001$ \\
\hline Hypertriglyceridemia $^{\circ}$ & $\mathrm{N}=5707$ & $1191(20.8)$ & $780(27.8)$ & $411(14.5)$ & $<0.001$ \\
\hline Mixed dyslipidemia $^{\circ}$ & $\mathrm{N}=5707$ & $935(14.8)$ & $598(21.3)$ & $337(11.9)$ & $<0.001$ \\
\hline DM2 ${ }^{\circ}$ & $\mathrm{N}=5568$ & $723(13.0)$ & $432(15.6)$ & $291(10.4)$ & $<0.001$ \\
\hline Obesity & $\mathrm{N}=5853$ & $1011(17.3)$ & $463(16.0)$ & $552(18.8)$ & $<0.01$ \\
\hline Metabolic syndrome ${ }^{\circ}$ & $\mathrm{N}=5660$ & $1306(23.1)$ & $629(22.3)$ & $677(23.8)$ & NS \\
\hline \multicolumn{6}{|c|}{ Cardiovascular events } \\
\hline Stable angina & $\mathrm{N}=5846$ & $113(1.9)$ & $68(2.3)$ & $45(1.5)$ & $<0.05$ \\
\hline Unstable angina & $\mathrm{N}=5846$ & $74(1.3)$ & $48(1.7)$ & $26(0.9)$ & $<0.05$ \\
\hline Myocardial infarction & $\mathrm{N}=5846$ & $180(3.1)$ & $154(5.3)$ & $26(0.9)$ & $<0.001$ \\
\hline CABG & $\mathrm{N}=5846$ & $46(0.8)$ & $39(1.3)$ & $7(0.2)$ & $<0.001$ \\
\hline PTCA & $\mathrm{N}=5846$ & $76(1.3)$ & $65(2.2)$ & $11(0.4)$ & $<0.001$ \\
\hline Transient ischemic attack & $\mathrm{N}=5846$ & $118(2.0)$ & $71(2.4)$ & $47(1.6)$ & $<0.05$ \\
\hline Ischemic stroke & $\mathrm{N}=5846$ & $41(0.7)$ & $25(0.9)$ & $16(0.5)$ & NS \\
\hline Claudicatio & $\mathrm{N}=5846$ & $82(1.4)$ & $73(2.5)$ & $9(0.3)$ & $<0.001$ \\
\hline Secondary prevention & $\mathrm{N}=5846$ & $501(8.6)$ & $354(12.2)$ & $147(5.0)$ & $<0.001$ \\
\hline LVH & $\mathrm{N}=5846$ & $526(9.0)$ & $288(9.9)$ & $238(8.1)$ & $<0.05$ \\
\hline \multicolumn{6}{|c|}{ Family history } \\
\hline Coronary heart disease & $N=5846$ & $681(11.6)$ & $323(11.1)$ & $358(12.2)$ & NS \\
\hline Cerebrovascular disease & $\mathrm{N}=5846$ & $454(7.8)$ & $205(7.1)$ & $249(8.5)$ & NS \\
\hline Dyslipidemia & $\mathrm{N}=5846$ & $1579(27.0)$ & $763(26.3)$ & $816(27.7)$ & $<0.001$ \\
\hline Hypertension & $\mathrm{N}=5846$ & 2897 (49.6) & $1346(46.4)$ & $1551(52.7)$ & $<0.001$ \\
\hline DM2 & $\mathrm{N}=5846$ & $1542(26.4)$ & $740(25.5)$ & $802(27.3)$ & NS \\
\hline
\end{tabular}

DM2: type 2 diabetes mellitus; CABG: coronary artery bypass graft surgery; PTCA: percutaneous transluminal coronary angioplasty; LHV: left ventricular hypertrophy; NS: not significant; ${ }^{\circ}$ Criteria used for specific diagnosis are described in Methods paragraph; ${ }^{\wedge}$ Criteria for definition are described in Methods paragraph; ${ }^{*}$ Men vs. women (non-parametric tests).

Table 4. CHD-risk classes and CVD-risk classes in total and sex stratified sample.

\begin{tabular}{|c|c|c|c|c|c|c|}
\hline & \multicolumn{3}{|c|}{ Framingham } & \multicolumn{3}{|c|}{ CUORE } \\
\hline & Total sample & Men & Women & Total sample & Men & Women \\
\hline Risk \%, mean $\pm \mathrm{SD}^{\circ *}$ & $10.1 \pm 8.3$ & $13.5 \pm 8.9$ & $7.0 \pm 6.3$ & $5.2 \pm 5.9$ & $7.8 \pm 7.1$ & $2.8 \pm 3.1$ \\
\hline \multicolumn{7}{|c|}{ Risk classes, $\mathrm{n}(\%)^{\S}$} \\
\hline$<5.0 \%$ & $1415(30.8)$ & $299(13.6)$ & $1116(46.6)$ & $2736(65.0)$ & $916(45.4)$ & $1820(83.2)$ \\
\hline $5.0 \%-9.9 \%$ & $1420(30.9)$ & $627(28.4)$ & $793(33.1)$ & $858(20.4)$ & $566(28.0)$ & $292(13.4)$ \\
\hline $10.0 \%-19.9 \%$ & $1257(27.3)$ & $884(40.1)$ & $373(15.6)$ & $470(11.2)$ & $403(20.0)$ & $67(3.1)$ \\
\hline$>=20.0 \%$ & $505(11.0)$ & 394 (17.9) & $111(4.6)$ & $142(3.4)$ & $126(6.6)$ & $8(0.4)$ \\
\hline
\end{tabular}

${ }^{\circ}$ Values calculated within the limits of each algorithm; ${ }^{*}$ Men vs. women (t-test) $\mathrm{p}<0.001$; ${ }^{\S}$ Men vs. women (non-parametric tests) $\mathrm{p}<0.001$. 
studies, while the DBP values resulted slightly lower (82 $86 \mathrm{mmHg}$ ) [18]. Compared to the population sample observed in the Atlante study, the CHECK cohort showed a higher overall incidence of hypertensive subjects $(52 \% \mathrm{M}$ vs. $45 \% \mathrm{~W})$, possibly due to a higher prevalence of older subjects in the CHECK sample.

Raised total cholesterol is a major cause of disease burden as a risk factor for ischaemic heart disease and stroke. Overall, it is estimated to cause 2.6 million deaths (4.5\% of total). The global prevalence of hypercholesterolemia among adults is about 39\% [13]. The mean plasma total cholesterol levels in the CHECK cohort were $205.3 \mathrm{mg} / \mathrm{dL}$. The prevalence of hypercholesterolemia was $55.6 \%$. Hypertriglyceridemia and mixed dyslipidemia were identified in $20.8 \%$ and $14.8 \%$ of the enrolled subjects, respectively. Lipid data observed in the CHECK and in the Atlante study showed relevant similarities: in both cohorts, women showed a higher level of TC, LDL-C, and HDL-C than the same level reported for men, but lower TG values. TC mean values observed in the CUORE population [22], on the other hand, were significantly higher than those found in CHECK (CUORE $225.4 \mathrm{mg} / \mathrm{dL} \mathrm{M}$ and $229.0 \mathrm{mg} / \mathrm{dL} \mathrm{W}$ vs. CHECK 202.5 $\mathrm{mg} / \mathrm{dL} \mathrm{M}$ and $208.0 \mathrm{mg} / \mathrm{dL} \mathrm{W}$ ). This difference is consistent with the trend of progressive reduction of blood $\mathrm{TC}$ levels observed over the last decades in Italy.

Impaired glucose tolerance and impaired fasting glycaemia are risk categories for future development of diabetes and cardiovascular disease [23]. A 2004 survey showed that more than 3 million people died from consequences of high blood sugar [24]. The global prevalence of diabetes is estimated to be $10 \%$ [13]. In the CHECK cohort, the mean level of fasting glucose was $98.3 \mathrm{mg} / \mathrm{dL}$ (101.7 M vs. $94.9 \mathrm{~W})$. Based on GPs diagnosis and/or presence of anti-diabetes therapies and/or plasma glucose levels $\geq 126 \mathrm{mg} / \mathrm{dL}$, type II diabetes prevalence was $13.0 \%$. This prevalence was higher than that observed in the Health Search GP's database of Italian general practice [25] for similar age $(10 \%-11 \%$; unpublished observation). This difference can be partially explained by different diagnostic criteria (based on at least two assessments in clinical practice compared to the single time determination in the CHECK study protocol), or by the identification of previously unknown cases in the CHECK sample, due to the generalised screening. In the Atlante cohort, the mean fasting glucose levels was lower $(93 \mathrm{mg} / \mathrm{dL} \mathrm{M}$ vs. $87 \mathrm{mg} / \mathrm{dL} \mathrm{W})$, leading to a lower prevalence of type II diabetes (Atlante $9 \% \mathrm{M}$ and $6 \% \mathrm{~W}$ vs. CHECK $15.6 \% \mathrm{M}$ and $10.4 \% \mathrm{~W}$ ). This difference could be partially explained by the different timeframe of the two studies (Atlante from 1998 vs. CHECK from 2001): over the last years, a generalised increase in the prevalence of type II diabetes in Western populations has been observed. Moreover, in the
Atlante study the blood glucose level was estimated from a sample of capillary blood, where glucose concentrations are on average significantly lower than in plasma.

Cardiovascular disease is the number one cause of death globally. In Italy, an estimated almost 240,000 people died from CVDs in 2008, representing $41 \%$ of all total deaths [26]. A clinical history of previous cardiovascular events was observed in $8.6 \%$ of the individuals included in the CHECK sample. The prevalence of such diagnosis was twice as high in men than in women. The prevalence of a clinical history of myocardial infarction was higher in the CHECK than in the Atlante study, especially in men (CHECK $5.3 \% \mathrm{M}$ and $0.9 \% \mathrm{~W}$ vs. Atlante $1.5 \% \mathrm{M}$ and $0.4 \% \mathrm{~W}$ ). The difference could be explained by the different methodological approach (information collected in a GP interview vs. hospital validation) and age differences.

The risk of future fatal and non-fatal cardiovascular events was assessed using two different algorithms, based on the outcomes of the Framingham and the Cuore cohorts. The mean coronary risk at 10 years, calculated with the Framingham algorithm, was $10.1 \%$, while the cardiovascular risk calculated with the CUORE algorithm showed an average value of $5.2 \%$. This difference was expected, since the Framingham algorithm is known to over-estimate the coronary risk of the Italian population [27]. Compared to the CUORE population data [28], the mean cardiovascular risk for the CHECK cohort was slightly lower (CUORE $7.8 \% \mathrm{M}$ and $2.8 \% \mathrm{~W}$ vs. CHECK $8.7 \% \mathrm{M}$ and $3.3 \% \mathrm{~W}$ ).

The innovative design of the CHECK study responded to the emerging need of determining the prevalence of cardiovascular risk factors directly from primary care, following a rigorous and unbiased research methodology. The randomisation of the sample, the web-based data collection of primary data, the territorial quality control operated by local coordinators, and the centralised laboratory procedures increase the external validity of the study, conferring a general significance to the epidemiological outcomes.

A follow-up of the cardiovascular events (fatal and nonfatal) occurred to all enrolled subjects is currently ongoing. It will provide the opportunity to evaluate the underlying prevalence of factors predisposing the onset of cardiovascular disease. Moreover, frozen samples provide the possibility of further biochemical evaluations, as the characterization of specific population subgroups through polymorphism search/genetic research.

The assessment of the gap between risk factor levels and recommended targets will provide evidence to aid clinical decision making about tailored non pharmacological and pharmacological interventions and to achieve optimal resources allocation.

The CHECK study has some methodological limita- 
tions, previously described. Importantly, the prevalence of cardiovascular events could have been underestimated, as the participating general practitioners reported those events at best of their knowledge, simply transcribing the diagnose related groups (DRG) codification of the events as reported by the hospital discharge letter, without any form of control on the appropriateness of the diagnoses. In addition, the peculiar design of the study, with progressive steps of GPs involvement, determined a participation rate lower than expected. The fact that local investigators did not participate in the enrolment meetings with the coordinating Centre, but were trained by their coordinators, may have weakened their motivation. However, no significant differences have been found between actively participating GPs and those who did not complete the preliminary steps.

\section{CONCLUSIONS}

The CHECK study confirmed the significant burden caused by the cardiovascular disease to the health care system and to society. In the randomised cohort observed, 1 out of 2 subjects was suffering from high blood pressure or high cholesterol, 1 out of 5 was obese and 1 out of 10 was a type 2 diabetic. The elevated prevalence of lifestyle risk factors observed in the Italian population raises the issue of moral hazard: the limited willingness of subjects covered by the National Healthcare System to take action and modify behavioural risks, in favour of deferring the issue to pharmacological prevention or treatment of the cardiovascular events at a later stage. While this normative issue is well known in economics of public health, the CHECK findings on the prevalence and co-occurrence of key behavioural and clinical risk factors is an emerging concern and it demands further research. Despite the fact that most subjects included in the study had been followed-up by general practitioners for years, the vast majority of them still showed the presence of one or more risk factors.

The high proportion of subjects with multiple risk factors in combination with the elevated prevalence of cardiovascular disease projects a shadow on the effectiveness of current cardiovascular prevention policies in primary care. The epidemiological outcomes of this study could be used as a baseline to set priorities and objectives for future intervention, as well as a benchmark, against which the effectiveness of the implementation of future prevention policies in primary care should be measured. The further development of the CHECK study is aimed to facilitate the recognition and to improve the management of cardiovascular diseases in the Italian general practice.

\section{ACKNOWLEDGEMENTS}

The CHECK study was supported in part by an unconditioned educa- tional grant from AstraZeneca SpA. Thanks to Maria Grazia Lanfranco and Elena Loggia for their assistance in completing our survey.

\section{REFERENCES}

[1] Alwan, A., Maclean, D.R., Riley L.M., et al. (2010) Monitoring and surveillance of chronic non-communicable diseases: Progress and capacity in high-burden countries. Lancet, 376, 1861-1868. doi:10.1016/S0140-6736(10)61853-3

[2] Fuster, V. and Kelly, B.B. (2010) Promoting Cardiovascular Health in the Developing World: A Critical Challenge to Achieve Global Health. Committee on Preventing the Global Epidemic of Cardiovascular Disease: Meeting the Challenges in Developing Countries. Institute of Medicine. National Academies Press, Washington.

[3] ERA (2009) Epidemiologia e Ricerca Applicata. http://www.atlantesanitario.it/index.php?option $=$ com con tent $\&$ view $=$ article $\&$ id $=82 \&$ Itemid $=93$

[4] ISTAT (2012) GeoDEMO. http://demo.istat.it/unitav/index.html?lingua=ita

[5] AIFA (2012) Rapporti OsMed-L'uso dei farmaci in Italia.

http://www.agenziafarmaco.gov.it/it/content/rapporti-osm ed-luso-dei-farmaci-italia

[6] CDC (2011) National Health and Nutrition Examination Survey. http://www.cdc.gov/nchs/nhanes.htm

[7] Adult Treatment Panel III (2001) Executive Summary of the Third Report of the National Cholesterol Education Program (NCEP) Expert Panel on Detection, Evaluation, and Treatment of High Blood Cholesterol in Adults. Journal of the American Medical Association, 285, 24862497. doi:10.1001/jama.285.19.2486

[8] Anderson, K.M., Wilson, P.W., Odell, P.M. and Kannel, W.B. (1991) An updated coronary risk profile. A statement for health professionals. Circulation, 83, 356-362. doi:10.1161/01.CIR.83.1.356

[9] AIFA (2012) Le nuove note CUF. BIF 5-6/2000. http://www.agenziafarmaco.gov.it/it/content/bif-5-62000

[10] Palmieri, L., Panico, S., Vanuzzo, D., et al. (2004) Evaluation of the global cardiovascular absolute risk: The Progetto CUORE individual score. Ann Ist Super Sanita, 40, 393-399.

[11] AIFA (2012) Le note. BIF 2/2004. http://www.agenziafarmaco.gov.it/it/content/bif-22004

[12] ISTAT (2012) 14 censimento generale della popolazione e delle abitazioni. http://dawinci.istat.it/MD/

[13] WHO (2009) Global health risks mortality and burden of disease attributable to selected major risks. World Health Organization, Geneva.

[14] WHO (2012) Tobacco. http://www.who.int/mediacentre/factsheets/fs339/en/inde x.html

[15] ISTAT (2012) Condizioni di salute e ricorso ai servizi sanitari: I fumatori in Italia. http://www3.istat.it/salastampa/comunicati/non_calendari o/20060110_00/ 
[16] Zuccaro, P., Pacifici, R., Giampaoli, S., et al. (2001) Fumo: Il contesto epidemiologico nazionale. Ital Heart Journal, 2, 13-18.

[17] WHO (2012) Physical inactivity: A global public health problem.

http://www.who.int/dietphysicalactivity/factsheet_inactiv ity/en/

[18] OEC (2004) Atlante italiano delle malattie cardiovascolari-Second edition. Ital Heart Journal, 5, 1S-101S.

[19] Finucane, M.M., Stevens, G.A., Cowan, M.J., et al. (2011) National, regional, and global trends in body-mass index since 1980: Systematic analysis of health examination surveys and epidemiological studies with 960 country-years and 9.1 million participants. Lancet, 377, 557567. doi:10.1016/S0140-6736(10)62037-5

[20] Baldissera, S., Campostrini, S., Binkin, N., et al. (2011) Features and initial assessment of the Italian Behavioral Risk Factor Surveillance System (PASSI), 2007-2008. Preventing Chronic Disease, 8, A24.

[21] Trinito, M., Bertozzi, N., Bietta, C., et al., (2006) Analisi di alcuni fattori di rischio cardiovascolari nella popolazione delle ASL partecipanti allo studio PASSI. Notiziario dell'Istituto Superiore di Sanità, 19, iii-iv.

[22] ISS (2012) Il Progetto CUORE: Assetto lipidico. http://www.cuore.iss.it/fattori/colesterolemia.asp

[23] Levitan, E.B., Song, Y., Ford, E.S. and Liu, S. (2004) Is nondiabetic hyperglycemia a risk factor for cardiovascular disease? A meta-analysis of prospective studies. Archives of Internal Medicine, 164, 2147-2155. doi:10.1001/archinte.164.19.2147

[24] WHO (2012) Diabetes. http://www.who.int/mediacentre/factsheets/fs312/en/

[25] Filippi, A., Vanuzzo, D. Bignamini, A.A., Sessa, E. Brignoli, O. and Mazzaglia, G. (2005) Computerized general practice databases provide quick and cost-effective information on the prevalence of angina pectoris. Ital Heart Journal, 6, 49-51.

[26] WHO (2011) Noncommunicable diseases country profiles. World Health Organization, Geneva.

[27] Menotti, A., Puddu, P.E. and Lanti, M. (2000) Comparison of the Framingham risk function-based coronary chart with risk function from an Italian population study. European Heart Journal, 21, 365-370. doi:10.1053/euhj.1999.1864

[28] ISS (2012) Il Progetto CUORE: Rischio cardiovascolare globale assoluto.

http://www.cuore.iss.it/fattori/RischioGlobaleAssoluto.asp 


\section{Appendix}

\section{Participating Investigators in the CHECK Study Group (Bold Type for Coordinators)}

Aalders Maria Anna

Abbate Giuseppe

Agati Riccardo

Alano Raffaele

Alba Mauro

Alemagna Silvia

Alunni Massimo

Alvaro Antonio

Amato Fabio

Ammendola Erminia

Amodeo Vincenzo

Amoretti Giovanni

Andrani Alberto

Antiga Ivo

Appolonia Giorgio

Aramini Enrico

Arisi Marco Emilio

Artebani Adriano

Atzei Massimiliano

Azzolini Micheline

Bachetti Francesco

Bagagli Franco

Baldicchi Lorella

Banzi Roberta

Barba Ettore Maria

Barbato Pasquale Claudio

Barral Gino

Battaggia Alessandro

Battigelli Doriano

Baudi Marina

Bellumori Giovanni

Beltrami Giuseppe

Benincasa Anna Maria

Berardi Mario

Berlengiero Claudio

Bernardelli Stefano

Bernardi Giuseppe

Bertelle Evandro

Bettini Gianluca

Bevacqua Giuseppe

Bevilacqua Stefano

Bianconi Giuseppe

Biggioggero Giovanni

Bini Vincenzo

Bocchino Giancarlo

Boccone Nicolfranco

Boito Giancarlo

Bollo Alberto Maria

Boncompagni Salvatore

Bond Giuseppe

Bonesi Maria Grazia

Bono Gianfranco

Boscaro Federica

Bossi Paolo

Bozza Giulio

Bracone Enrico

Brandodoro Lucio

Brasesco Pierclaudio

Breviario Adele

Brizzi Antonio

Brugnetta Maurizio

Bruno Giuseppe

Buemi Giuseppe

Bufano Carmine

Bugli Tiziano

Burigo Daniela
Buzzatti Agostino

Caccamo Orazio Antonio

Cadamosti Danilo

Cagliesi Francesco

Caleffa Manuela

Cammisa Nicolo'

Campo Franceso

Campobello Margherita

Caputo Stanislao

Caraccio Nicola

Cardi Silvio

Cardinale Fulvio

Caregnato Massimo

Cariola Gianni

Carlino Saverio

Carminati Luisa Angela

Carnelli Feliciano

Carnesalli Franco

Caruso Ciro

Casale Ezio

Casini Marcella

Cassanelli Marco

Castiello Maria Luisa

Castriotta Antonio

Catalano Domenico

Cataldi Maria Elvira

Ceccarini Agostino

Celebrano Mario

Celora Amedeo

Cerracchio Alessandro

Cesaro Andrea

Cesaro Federico

Chiriatti Alberto

Cipriani Rosa

Collura Giuseppe

Colombo Valter

Coluccia Salvatore

Conte Sergio

Corda Andrea

Costa Roberto

Cottani Antonio

Crivellenti Giuseppe

D’Ambrosio Gaetano

D'Angelo Massimo

Dalla Rosa Rosanna

Damico Giansanto

De Andreis Bessone Pier Luigi

De Benedictis Antonio

De Conto Umberto

De Mola Cosimo

De Rosa Antonio

De Tommasi Roberto

Del Nero Barbara

Della Briotta Ivana

Dell'Orco Mario Domenico

Dell'Orco Mario Lucio

Di Candia Giuseppe

Di Carlo Vittorio

Di Febo Enrico

Di Feo Antonio

Di Fraia Giovanni

Di Fulvio Aristide

Di Nardo Dionisio

Dolmetta Franco

Donzelli Luigi

Dughiero Fausto
Durando Andrea

Ercolino Luigi

Fabbri Stelania

Fabrizio Nicola

Falchi Raffaello

Fariello Ciro

Fascendini Emilvio

Fasulo Serenella

Federici Laura

Ferioli Paolo

Ferrari Vincenzo

Fidelbo Melchiorre

Filetti Giuseppe

Filippini Giovanni

Fogher Michele

Franchini Carlo Andrea

Frascati Angelo

Frignani Patrizia

Fronteddu Pier Francesco

Gadaleta Caldarola Gennaro

Galantini Giovanna

Gallicchio Nicola

Gallina Franco

Gallo Silvano

Gambino Fortunato

Gambuzza Guglielmo

Garaffa Elio

Garagiola Alberto

Garofalo Remigio

Garrone Alfonsine

Gatta Luigi

Gennari Massimo

Gerace Antonio

Geremia Maria Alessandra

Germini Fabrizio

Giacci Luciano

Giannini Olivia

Giordano Stefano

Giovannelli Umberto

Giuffré Giuseppe

Giunti Giuliana

Glaviano Bruno

Gorletta Giovanni

Grand Paola

Grassini Giovanni

Grasso Anna Maria

Grasso Giuseppe

Grasso Maria Filomena

Greco Agostino

Grifagni Marcello

Grilli Piero

Grimaldi Emanuela

Grimaldi Nicola

Grosso Marco

Guarnera Lucia

Guerra Antonio

Guillaro Bruno

Gussoni Barbara Rita

Ianiro Gabriella

Ilardi Salvatore

Imbalzano Pasquale

Inguscio Cherubino

Invernizzi Giovanni

Iocca Tommaso

Kos Egidia

La Mattina Rosolino
La Torre Angelo

Lacava Cosimo

Lalli Pasqualino

Lamera Giorgio

Lanza Gerardo

Lardo Gerardo

Laringe Matteo

Lattanzio Giuseppe

Le Foche Luca

Leo Rosanna

Leuzzi Giacomo

Lipari Antonino

Lipari Francesco

Lippa Luciano

Lo Conte Maurizio

Lo Giudice Domenico

Lonati Rossella

Lorenzina Enrico

Magi Lorenzo

Magliozzo Francesco

Mallamo Luciano

Mantovani Licia

Marcenaro Alessandro

Marchetti Anna Rosa

Mariano Carlo

Marino Antonino

Mariuz Manuela

Maroni Achille

Martori Ampelio

Masoch Gigliola

Mattioli Carlo

Mattioli Mauro

Maurici Vincenzo

Mauro Nicola

Mazzardi Lidia

Mazzi Wainer

Mazzocchetti Alvaro

Mazzoleni Francesco

Mazzorana Michela

Medagliani Giorgio

Medea Gerardo

Merlino Giovanni

Merone Laura

Metrucci Antonio

Mezzano Silvio

Micchi Alessio

Micheli Pietro Severo

Milazzo Vito

Minafra Francesco

Minetti Luca

Mirandola Cipriano

Monari Gianluigi

Mongiello Claudio

Montano Giovanni

Montera Carmine

Moretti Marino

Morgana Ignazio

Morganti Mauro

Mormile Annunziata

Moro Roberto

Mostacciolo Francesco

Mourglia Danilo

Murari Tiziana

Muratore Alessandro

Murgia Rosalba

Naccari Massimo 


\author{
Napoli Luigi \\ Nardacci Giuseppe \\ Nebiacolombo Cristina \\ Negri Fabrizio \\ Nicolini Gianfranco \\ Nigro Antonio \\ Noia Emanuela \\ Nuti Claudio Pietro \\ Olivani Enrico \\ Orlando Celestina \\ Padovan Letizia \\ Padula Maria Stella \\ Pagan Maurizio \\ Pannacci Valerio \\ Pantalone Vincenzo \\ Paolini Italo \\ Papandrea Giampaolo \\ Papini Giovanni \\ Papulino Francesco \\ Paradisi Enza \\ Parisi Carmela \\ Parretti Damiano \\ Pasculli Domenico \\ Pasinelli Pietro Carlo \\ Pasqualetto Salvatore \\ Passamonti Marco \\ Passaro Vincenzo \\ Pederzani Fabio \\ Pedrazzoli Giuliano \\ Pelizzari Pier Carlo \\ Pernici Pierrenato \\ Pesaresi Carlo \\ Pesce Gian Luigi \\ Petrucci Marco \\ Petrucci Mauro \\ Petrulli Carmela \\ Petti Stefano \\ Piccinocchi Gaetano \\ Picciotto Rinaldo \\ Piccolo Francesco \\ Pierobon Ivo \\ Pilone Rita
}

Piva Roberto

Pizzillo Carlo

Plebani Franco

Polistina Stefano

Pontari Antonino

Poppi Maria Cristina

Portanti Carla

Prencipe Giovanni

Prestifilippo Alessandro

Procopio Antonio

Profeta Gaetano

Proietti Carlo

Quattrocchi Pietro

Raciti Teodoro

Ragazzoni Anna

Rattini Emanuela

Reale Emanuela

Redaelli Dario

Reggiani Claudio

Ricotta Giuseppe

Rigamonti Rodolfo

Righini Velella

Rinaldi Vanna

Rista Pierangela

Romano Salvatore

Romei Federico

Rossi Alberto

Rossi Angelo

Rossi Francesco

Rossi Gianluca

Rosso Lucia

Rovazzani Massimo

Rovelli Monica

Rovescala Pietroclaudio

Rubicini Giuseppe

Rubini Stefano

Russo Carolina

Russo Vincenzo

Sala Massimo

Salurso Daniele

Salvaderi Maria Dionice

Salvato Alberto
Salvetti Andrea

Salvio Giuliano

Samani Fabio

Sammarco Renato

Santoiemma Luigi

Santoro Michele

Sassarini Graziano

Savino Andrea

Scaglione Matteo

Scarano Libero

Schiavone Ciro

Scola Vincenzo

Scorpiniti Anna

Scotto D'Antuono Antonio

Scovotto Maria Antonietta

Scuri Maurizio Giovanni

Scuteri Antonio

Sebastianelli Giuliano

Sebben Maurizio

Sforza Pasqualino

Sfragara Ignazio

Sicari Giuseppe

Simonini Giorgio

Soldani Miriam

Soverina Patrizio

Spagnolo Beatrice

Sperandio Massimo

Spezzano Alfredo

Steri Lia

Storni Paolo

Strada Sonia

Stramenga Carlo

Tagliabue Paola Fausta

Tarabini Legnoaura

Tarallo Nicola

Tei Alessandro

Tei Gian Paolo

Testi Sergio

Testolin Ennio

Tibo Angela

Titone Nicolò

Tomasello Antonino
Tondi Lidia

Torti Giorgio Tommaso

Toscano Emanuele

Tota Maria Fiorenza

Tozzoli Alfonso

Travaglini Rita

Trois Paolo

Trotta Gaetano

Tuia Bruno

Turbil Enrico

Ughetti Claudio

Urru Cesare

Valdevit Maria

Valente Fabio

Valenti Marco

Valle Lucia

Valletta Domenico

Valore Salvatore

Varriale Antonio

Varrica Gaetano

Ventriglia Giuseppe

Venturelli Antonio

Vesco Giuseppe

Vezzosi Angelo

Viola Dario

Viscusi Bruno

Vita Salvatore

Vitali Franco

Vittozzi Dante Sergio

Vivona Giacomo

Volpe Augusto

Volponi Damiano Antonio

Voza Italo

Zadra Alessandro

Zaninetti Piero

Zanini Riccardo

Zennaro Walter

Zingaro Angelo

Zito Alfonso

Zollino Luciana

Zovi Maria Carla

Zunino Roberto 